\title{
BMJ Open Qualitative research on the work experience of pharmacists in fever clinics and isolation wards of designated hospitals for novel coronavirus pneumonia (COVID-19)
}

Jie Hao ํㅜ, Xiaojuan Wang, Xuedong Jia, Yantao Yang, Shuzhang Du, Zhao Yin

To cite: Hao J, Wang X, Jia X, et al. Qualitative research on the work experience of pharmacists in fever clinics and isolation wards of designated hospitals for novel coronavirus pneumonia (COVID-19). BMJ Open 2021;11:e048466. doi:10.1136/ bmjopen-2020-048466

- Prepublication history for this paper is available online To view these files, please visit the journal online (http://dx.doi org/10.1136/bmjopen-2020048466).

Received 29 December 2020 Accepted 22 0ctober 2021

Check for updates

(c) Author(s) (or their employer(s)) 2021. Re-use permitted under CC BY-NC. No commercial re-use. See rights and permissions. Published by BMJ.

Department of Pharmacy, The First Affiliated Hospital of Zhengzhou University, Zhengzhou, Henan, China

Correspondence to

Mr Zhao Yin;

yinzha00601@163.com

\section{ABSTRACT}

Objective To understand the work experiences of pharmacists in fever clinics and isolation wards of designated hospitals for the novel coronavirus pneumonia in China and provide the basis for work management strategies and psychological interventions in pharmacy for prevention and control of future epidemics.

Methods Using qualitative research methods, 13 pharmacists who met the inclusion criteria and worked in fever clinics and isolation wards of designated hospitals for novel coronavirus pneumonia attended focus group interviews and semistructured interviews. The Colaizzi analysis method was used for data analysis, summary and induction.

Results Three themes were identified: (1) the roles of pharmacists in fever clinics and isolation wards in epidemic prevention and control, including ensuring the supply of medicines, providing medication guidance for patients, providing medication information for physicians and nurses, and participating in infection control; (2) the difficulties at work, including the lack of office equipment, information equipment and other infrastructure, the difficulty of management of pharmacists in isolation wards, challenging environments, and insufficient attention of hospitals, medical staff, and society to pharmacists; (3) the loopholes in drug management, including the management of special-class drugs and national free AIDS antiviral drugs, and the retrieval of drugs.

Conclusion Pharmacists have played an essential role in the fight against the epidemic of novel coronavirus pneumonia in China. The themes and experiences identified in this study can offer guidance to the pharmacy society in implementing strategies to prepare for future public health events.

\section{BACKGROUND}

At the end of December 2019, unexplained mass pneumonia cases were found in Wuhan, China, which aroused health authorities' attention. On 7 January 2020, the pathogen was identified as a novel coronavirus (2019$\mathrm{nCoV}),{ }^{1}$ and the WHO officially named the disease COVID-19 on 11 February 2020. The
Strengths and limitations of this study

- The strength of this study was using qualitative methodology to find the work experiences of pharmacists who participated in the work of fever clinics and isolation wards in the fight against COVID-19.

- This study only included participants from pharmacists, thus fully representing the views of the pharmacists' profession.

- The study was performed from a constructivist point of view using an interpretative phenomenological epistemology.

Both focus interviews and semistructured interviews were conducted.

- The results may be limited in the region with pharmacists' recruitment and may not be generalisable across China.

disease is highly contagious, and people of all ages and genders are susceptible to COVID19. Moreover, it has recently been found that patients with asymptomatic or mild symptoms have a very high transmission capacity, and that spread can occur early in the infection process. ${ }^{2}$ The prevention and control of COVID-19 is a worldwide public health problem $^{3}$ and the WHO has declared the current epidemic to be a global pandemic. ${ }^{4}$ At present, China's epidemic prevention and control have achieved a strategic victory, ${ }^{5}$ but the pandemic spread in the world is still rapidly increasing. ${ }^{6}$ In the face of a severe and complex international epidemic, it is worth summarising China's anti-epidemic experience.

During the prevention and control of the epidemic, physicians and nurses on the front line are experiencing heavy work pressure and mental stress. Many studies have explored the experiences and feelings of these physicians ${ }^{7}$ and nurses. ${ }^{89}$ The outbreak of COVID-19 has 
dramatically affected the mental health of medical staff. ${ }^{10}$ Pharmacists are an essential part of the healthcare system. They are responsible for drug supply, drug management, and pharmaceutical services in epidemic prevention and control. ${ }^{11}$ Pharmacists have been working hard on the front line. One study ${ }^{12}$ investigated the symptoms of work burnout and post-traumatic stress in community pharmacists during the COVID-19 pandemic and how the adaptability of pharmacists may prevent them from burnout and reduce the incidence of mental illness. ${ }^{13}$ However, there are only a few studies on the work experience of hospital pharmacists during COVID-19. ${ }^{10}$

After decades of development, the Chinese hospital pharmacy system has made significant progress. However, it is unclear whether Chinese hospital pharmacists can stand the test of the epidemic or not. In China, when a person has a fever and is suspected of infection with COVID-19, they must go through a fever clinic for a preliminary examination. A fever clinic is a special clinic set up by the hospital outpatient department to prevent and control acute infectious diseases. 'Fever pharmacy' is a pharmacy-only service for fever clinics. The primary job responsibilities of the pharmacists of the fever pharmacy were dispensing. In this study, we aimed to adopt qualitative research methods to deeply explore pharmacists' work experience in fever clinics and isolation wards in the fight against the epidemic. The study was performed from a constructivist point of view using an interpretative phenomenological epistemology. Based on the notion that social phenomena are constructed by the communal making of meaning about the underlying phenomena, we aimed to construct insightful accounts of pharmacists' work experiences. The results can provide a theoretical basis for pharmacists' work management strategies and psychological interventions in future epidemic prevention and control.

\section{METHOD \\ Participants}

The Sixth People's Hospital of Zhengzhou (SPHZ, 420 beds with 35 designated COVID-19 treatment beds) and the First Affiliated Hospital of Zhengzhou University (FHZU, 8500 beds with 60 COVID-19 treatment beds) are the designated hospitals for treating patients with COVID-19 in Zhengzhou, Henan Province. SPHZ, also known as the Henan Provincial Hospital for Infectious Diseases, mainly treated patients with mild COVID-19. In contrast, FHZU mostly treated critically ill patients. The investigators used the purposive sampling method and selected pharmacists at these hospitals as study subjects. The inclusion criteria were pharmacists (1) who had worked in fever clinics or isolation wards, (2) who had participated in the anti-epidemic work for 1 week or more, and (3) who were willing to participate in this study. The sample size was determined based on the saturation principle, and 13 subjects were finally included.

\section{Study design and data collection}

A phenomenological method was used to conduct focus interviews and semistructured in-depth interviews with pharmacists working at the two hospitals. Focus groups share many common features with less structured interviews, but there is more to them than merely collecting similar data from many participants at once. A focus group is a group discussion on a particular topic organised for research purposes. This discussion is guided, monitored and recorded by a researcher. ${ }^{14}$ Semistructured interviews consist of several key questions that help define the areas to be explored and allow the interviewer or interviewee to diverge from pursuing an idea or response in more detail. ${ }^{14}$ Due to the restricted conditions during the pandemic, a focus group interview was conducted in SPHZ, then semistructured interviews were conducted at FHZU.

Written consent was obtained from study participants. Interviews were conducted through video and face-to-face interviews. The interviews were held from April 2020 to May 2020, and each session ranged from 15 to $60 \mathrm{~min}$. All interviews were audio-recorded, and the non-verbal behaviours of the interviewees were recorded in writing. After preinterviews with two interviewees, the interview outline was finally determined as follows: (1) After experiencing this fight against the epidemic, please talk about your overall work experience; (2) In the future fight against epidemics, what do you think you can do to reflect better professional values?; (3) In this fight against the epidemic, what difficulties have you encountered? What positive experiences have you encountered? Would you please talk about how you experienced them at that time? Investigators also collected demographic information from the interviewees.

\section{Data analysis}

Within 24 hours after the end of the interview, investigators transcribed the recordings word-by-word. Study subjects were coded as P1-P13. During the transcription process, investigators wrote a memo and reflection diary and continuously adjusted the interview strategy in the follow-up sessions to improve the research quality. Two investigators independently completed the data analysis, coding and thematic analysis. Group discussions were conducted to form a unified opinion. The Colaizzi 7-step analysis method ${ }^{15}$ was used to analyse the transcription data.

The main steps are briefly described as follows: (1) read all interview records repeatedly and carefully; (2) analyse the meaning of the records in depth and select the statements of significant importance; (3) code recurring and meaningful opinions; (4) collect the coded opinions to form a theme; (5) write a detailed and complete description and present it to the interviewee; (6) identify similar opinions and sublimate the theme concept; (7) confirm the authenticity of the interview content with the interviewee. 


\begin{tabular}{|c|c|c|}
\hline Characteristics & Frequency (\%) & Mean \pm SD \\
\hline Age & & $33 \pm 3.2$ \\
\hline \multicolumn{3}{|l|}{ Gender } \\
\hline Male & $5(38.46)$ & \\
\hline Female & $8(61.53)$ & \\
\hline \multicolumn{3}{|l|}{ Hospital } \\
\hline \multicolumn{3}{|c|}{$\begin{array}{l}\text { The Sixth People's Hospital of } 5 \text { (38.46 ) } \\
\text { Zhengzhou }\end{array}$} \\
\hline $\begin{array}{l}\text { The First Affiliated Hospital of } \\
\text { Zhengzhou University }\end{array}$ & $8(61.53)$ & \\
\hline Working years & & $8.46 \pm 3.07$ \\
\hline \multicolumn{3}{|l|}{ Education } \\
\hline Undergraduate & 10 (76.92) & \\
\hline Master & $3(23.07)$ & \\
\hline \multicolumn{3}{|l|}{ Job title } \\
\hline Pharmacist & $8(61.54)$ & \\
\hline Pharmacist-in-charge & $5(38.46)$ & \\
\hline \multicolumn{3}{|l|}{ Voluntary } \\
\hline Volunteer & $10(76.92)$ & \\
\hline Appointed by the hospital & $3(23.07)$ & \\
\hline
\end{tabular}

\section{Patient and public involvement}

Neither patients nor the public were involved in the design, conduct, report or dissemination of our research plan.

\section{RESULTS}

The average age of the study subjects was 33 years old, with $61.53 \%$ female. They have an average of 8.46 years of work experience. The demographic information is shown in table 1. Three themes were extracted through the transcription, coding and interpretation of the interview data.

\section{Theme 1: the epidemic promotes pharmacists' expanding scopes of practice under emergency conditions \\ Theme 1.1: expand the stock and use of non-regularly stocked medications}

Since the outbreak of COVID-19, the National Health Commission and Tongji Medical College of Huazhong University of Science and Technology have successively issued COVID-19 diagnosis and treatment plans. The guidelines are currently in their seventh update. The guidelines included treatment drugs not routinely stocked in hospitals, such as arbidol and lopinavir/ritonavir. Pharmacists working in fever clinics and isolation wards were in charge of guaranteeing the supply of these medicines. This was the most urgent task for a pharmacist in an epidemic situation. During the outbreak of COVID-19, drugs had been supplied in good order.

First of all, I must guarantee the supply of medicines. Then, I take over this fever clinic as a team leader, schedule the time, endure the supply of medicines for medical staff, prepare medications and handle other emergencies. I received a notice from the Drug Dispensing Department that night to prepare drugs for the fever pharmacy. (P11)

In the early stages of the COVID-19 pandemic in China, traditional Chinese medicine (TCM) pharmacies provided Chinese herbal medicine decoctions to healthcare providers and patients in isolation wards. These TCM medications were used as part of comprehensive treatment modalities for patients with COVID-19.

\section{Theme 1.2: provide medication guidance and psychological} support for patients

Pharmacists began to provide pharmaceutical care, such as prescription reviews and medication counselling for patients in fever clinics or isolation wards. Although these pharmaceutical activities are routine responsibilities for pharmacists, ${ }^{16}$ it is challenging for pharmacists to do that when wearing heavy protective clothing and their need to overcome psychological barriers and practical difficulties during the epidemic.

The fever clinic is like a small hospital. I have to do everything by myself, including reviewing doctor's orders, double-checking, and explaining medications to others. It is a whole process. $(\mathrm{P} 7)$

The patient (after obtaining the medicine) did not know anything. He brought it over and asked me how to take it. He could not bring the prescription with him, so he came over and asked me how to take this medicine. Sometimes I would write to him on the medicine box how to take it. I would tell him when to take it, for example, before or after the meal. I told him what symptoms he might have when he took medicine, or how often he should take medicine, what adverse reactions he should pay attention to, and if there were any such reactions; he should stop taking medicine and call the hospital. (P12)

COVID-19-positive or suspected patients were in a state of extreme panic and helplessness when they saw physicians in the hospital and faced tremendous psychological pressure in the early stage of the outbreak. Pharmacists could provide them with psychological comfort and guidance at the dispensing window.

Not much professional value can be embodied. However, there may be a little bit of comfort for the patient's psychology. Explain to them the medication and relieve their stress a little bit. Then I think this may be more important. (P8)

Theme 1.3: increasing medication consulting services for medical staff

In the fever clinics, pharmacists, physicians and nurses were fighting together on the front line. During the work process, pharmacists strengthened communication with physicians and nurses and expanded pharmaceutical care. Occasionally, they would provide physicians and 
nurses with medication suggestions. They also shared experiences with physicians on the use of medicines for COVID-19.

At first, only physicians and nurses from the respiratory department and infectious diseases department were involved, and some were transferred from the internal medicine department. When it comes to pediatric medicines or other medicines or dosages, they had no idea. They would consult you sometimes on that. (P8)

Our department also had some medicines that were distributed in the group to fight the epidemic. Those comparisons, and some precautions, as a whole, including some forms, were all sent to them (medical staff). (P13)

Theme 1.4: actively participating in epidemic prevention and control management of fever clinics

Under normal circumstances, hospital pharmacists are rarely involved in nosocomial infection control in China. However, pharmacists have mentioned their full participation in infection control, including (1) disinfecting fever pharmacies, (2) complying with relevant infection control processes, and (3) transforming the work practice and strictly executing work procedures under epidemic prevention. To avoid cross-infection, the disinfection work in the fever clinics was done by the pharmacists themselves, which was a significant challenge for them.

Because a pharmacy is an independent unit, both the pharmacy hygiene and disinfection you have to do by yourself, and then every morning you go to work, and every night after the night shift, you should mix chlorhexidine with water at a ratio of 1:2000, then use this mixture to disinfect the unit and mop the floor or something. (P9)

Fever clinics were divided into contaminated areas, semicontaminated areas and clean areas. Regulations on pandemic prevention and control should be rigorously followed by all personnel working at fever clinics. None of the two hospitals had reported a single case of COVID-19 infection among healthcare providers.

There is a standard protection process. There are ultraviolet lamps in every transition area, and these areas are disinfected regularly every day. (P10)

The work mode of the pharmacists in the isolation wards also played a crucial role in infection control. In the isolation wards, the medicines were generally obtained without contact, which avoided cross-infection.

The pharmacist and the nurse getting the medicine don't meet each other. The nurses stay in the inner room. Then we will put the medicine in the designated location, and they are there. Then after our pharmacist is gone, the nurse inside can come out and get the medicine. (P5)
In the isolation wards, they have a special medicine basket. Hmm, the kind of medicine basket and the box. Then they prepare and disinfect it or something. Then the caregiver comes over to get the basket. After we get the prescription, we also designate one person to get the medicine and the other to check it. After we check it right, we give it to them. Then the caregiver takes the basket up and hands it to the nurse. (P9)

\section{Theme 2: difficulties and challenges pharmacists encountered} during the epidemic

Before COVID-19, we had the experiences of SARS, H1N1 and other epidemics in China. This allowed us to carry out measures such as isolation and treatment in an orderly manner. However, the novel coronavirus is a new type of virus. Many things are fumbled along the way in our work, and problems need to be solved urgently. The main difficulties encountered were as follows:

\section{Theme 2.1: lack of infrastructure for temporary workplaces}

At the beginning of the epidemic, the fever clinic pharmacy was remodelled from a sundries room in the fever clinic. Hospitals were in a state of panic, and pharmacists had to find their way to set up essential workplaces at that time.

The fever clinic pharmacy was established in a rush, and then there were some things that needed to be communicated. Well, we couldn't get it right soon. The tasks were heavy, we asked it urgently, and finally, we got it quickly. So, at that time, it was difficult to meet our requirements. (P11)

Theme 2.2: insufficient supplies of personal protective materials At the start of the epidemic, the entire society faced insufficient supplies of anti-epidemic items, as did the pharmacists in the fever clinic.

As for the protective materials, they are all managed by the fever clinics (if we need the materials); ask the head nurse. But there is still a small episode, for some supplies, even the head nurse cannot get them, such as gowns, masks, gloves, goggles, and this kind of things. (P11)

\section{Theme 2.3: lack of workers}

Although pharmacists in the isolation wards did not directly contact patients and medical staff, they were prone to panic due to their proximity to the isolation wards, which also caused specific difficulties in their management.

Some pharmacists are too alert. Because they are too sensitive, they are against some arrangements in work. It is difficult to arrange some tasks. To tell him that, we have all done the disinfection, there is no problem; there should be no problem. Because you 
are on the second floor, you are not so much (risky). But there are still concerns. (P11)

\section{Theme 2.4: tough living conditions}

When the fever clinic pharmacy was initially set up, the environment was harsh. It was also challenging and not very convenient to take a bath or go to the toilet during working time. Furthermore, there was a lack of sunlight for a long time, which caused a weakened immune system. Pharmacists had to overcome these difficulties of harsh living conditions at work.

There were a lot of people. It was inconvenient to take a bath. Everyone had to line up. In addition, as your N95 mask was strangled, your face got hurt. But we felt OK. But they didn't give us N95 masks anymore. Everyone put on ordinary masks, so it was nothing. (P6)

\section{Theme 2.5: insufficient attention to pharmacists}

Pharmacists found that not much attention was paid to them in any aspect by physicians, nurses, administrators, social media and the public. This made pharmacists feel that their value in fighting the COVID-19 epidemic was not fully recognised. The following pharmacists explicitly mentioned the negative work experience during interviews. Pharmacists ensured the supply of medicines and the medication safety of patients in a fever pharmacy, but physicians and nurses still ignore the role of pharmacists.

Anyway, when they were chatting with you, they thought, why were you here? They would ask you, did you know? They would ask the pharmacists, why were you pharmacists here? At that time (the epidemic) was much better. Then they would say, why didn't you go home like those cashiers? (P12)

The staff working in fever clinics were divided into batches and changed every 2 weeks. To prevent crossinfection, staff members in the previous batch would be isolated together, but hospital administrators sometimes ignored the isolation of pharmacists.

(Without isolation) If something happened to me, if I was infected with this disease, I might die, I might die without regret, I participate voluntarily. But if I was because of this (no isolation), I went back and infected my family, father, and mother. I feel ashamed. (P12)

Most people, including those on social media, only saw the hard work of physicians and nurses. They rarely praised the pharmacists for their work.

Many benefits, including tickets to scenic spots, were nothing to do with pharmacists in many times. They were for physicians and nurses. I saw the news one day that only one scenic spot, I forgot which one, mentioned physicians, nurses, and pharmacists. There were a lot of comments below. Finally, one of the scenic spots talked about pharmacists. (P11)

\section{Theme 3: challenges in drug management procedure during outbreaks}

Medications in the isolation wards were generally only allowed to come in but not come out, which challenged the management of controlled substances, national free antiviral drugs (referred to free drugs thereafter) and drug retrievals from the isolation wards. However, during the epidemic outbreak, especially in the early stage, the hospital managers did not take appropriate measures to deal with drug management, which led to many loopholes in drug management.

\section{Theme 3.1: difficulty to standardise special-class drug management}

Special-class drugs in China have controlled substances. These drugs have potent analgesic and sedative effects and are essential drugs for clinical diagnosis and treatment. However, irrational use is prone to dependence and addiction. If they are not managed carefully, they may flow into illegal channels, which will cause serious social harm. Medical institutions at all levels should strictly control the management of special-class drugs. The COVID-19 epidemic is a public health emergency, and initially, there were no clear procedures for managing these medications. Unfortunately, this has led to difficulties in managing their clinical use. The prescription management measures for controlled substances in China have strict prescription signatures, stamps and retention requirements. These requirements could not be met during the epidemic and could only be rectified after the epidemic.

A prescription for controlled substances is required, a red prescription, and a planned prescription. How could you get this prescription?... But now we don't take it back; we just take a photo to keep it. (P1)

We were distributing medicines that were not in compliance with the regulations. If we asked for something from them, we could not send the medicines to them. They had nothing, no prescription, just electronic messages, and no signatures. There were no handwritten signatures. (P11)

A more severe management loophole exists in the recycling of empty ampoules. After the controlled substances were used up, empty ampoules could not be recovered promptly. It makes it difficult for pharmacists to monitor the use of controlled substances on time, which can cause serious problems such as drug abuse.

There were also empty ampules, which I could not collect either. The leader asked us to take photos after destroying them. This was the last method. This could only be done in special periods. We were groping the way, and loopholes definitely existed. (The loophole was) Have they (the nurses) broken the 
ampule? This was possible that they took a photo after destroying an empty ampule that day. The next day they might take a photo of the same empty ampule from a different angle. (P1)

\section{Theme 3.2: difficulty retrieving drugs according to the normal procedure}

Generally, when a patient refuses or the therapeutic schedules are changed, dispensed drugs need to be returned to the pharmacy. However, this standard practice could not be implemented from isolation wards, resulting in potential risks.

(The drugs) were all piled up in the isolation wards, and we finally got them back together. There were refrigerators (there), but some of them did not pay much attention. As you know, we should remind the nurses of which ones to be put in the refrigerators. Some nurses might not pay attention. And some nurses who used to get medicines frequently before, and some nurses did not usually come in contact with medicines, so they did not know how to put. (P2)

Theme 3.3: lack of management protocols for national free AIDS antiviral drugs during the epidemic

Lopinavir/ritonavir is a prescription drug strictly controlled and provided to patients with AIDS free of charge according to AIDS Prevention and Control Regulations in China. In the epidemic, there were no specific medicines for COVID-19. The Chinese guidelines recommended a trial of lopinavir/ritonavir. However, there were no established protocols on the use, storage and accounting management under this circumstance.

Maybe it was negligent at first. What was the first problem? The number of tablets in every bottle was unchanged. If a few tablets fell on the ground after the bottle was opened, I could know this, and the head nurse would report the accident to me. (P11)

A problem occurred when these drugs were recovered, that is, the clinical use of these drugs, in other words, the record of these drugs, was incomplete. Because lopinavir-ritonavir was only described in the physician's order, and there was no more information. No detailed description for some patients, and there was no record in the case. Therefore, the nurses used lopinavir-ritonavir directly. So it was challenging for you to find those records later. (P11)

\section{DISCUSSIONS}

This study was the first qualitative research to deeply explore pharmacists' work experiences in fever clinics and isolation wards at the designated COVID-19 hospitals. In the fight against COVID-19, physicians and nurses have direct patient contact, and the public recognise their vast contributions and sacrifices. ${ }^{17}$ Unfortunately, our investigation revealed that most pharmacists' work is behind the scenes. Their assistance and sacrifices may be easily ignored and quickly forgotten by the public. Data showed that as of 8 April 2020, more than 450 pharmacists had been infected with COVID-19 globally. ${ }^{18}$ Therefore, our research attempts arose to draw attention to the pharmacists' critical role in the fight against COVID-19 and the hardships they suffered. Our findings help provide references for future pharmacists for responding to similar public health events.

\section{The expanded roles of pharmacists in the fight against the COVID-19 epidemic}

During the epidemic, we saw pharmacists' expanded roles in fever clinics and isolation wards, from ensuring medication and disinfectant supplies to providing drug information to physicians or nurses and conducting education for patients. The results are similar to previous studies ${ }^{19-22}$ Pharmacists have played an essential role in epidemic prevention and control. ${ }^{22}$

Compared with hospital pharmacists in China, pharmacists in other countries pay more attention to patients with chronic diseases ${ }^{23}$ aiming to achieve better drug management. ${ }^{24}$ In many countries, including the UK, pharmacists in hospitals are engaged in tasks beyond their regular mandate, such as developing and updating their skills in intensive care, directly managing patients with COVID-19, providing support to the service hotline and becoming a part of the national emergency team. ${ }^{18}$

\section{Difficulties encountered by pharmacists at work}

Pharmacists have felt the distress of not being taken seriously by the medical team and the public to fight against COVID-19, and the finding is consistent with the results of studies in other regions. ${ }^{22}{ }^{25}$ Studies have shown that $36 \%$ of pharmacists surveyed in Chongqing believe that hospital leaders did not pay (or did not pay enough) attention to pharmacists. Around $17.7 \%$ of pharmacists believe medical staff do not recognise or seriously recognise pharmacists. ${ }^{26}$ There are many reasons for this to occur. For a long time, China has emphasised physicians over medicines, and pharmacists have received insufficient attention. ${ }^{27} 28$ The public are also not highly aware of the work of pharmacists. They believe that the pharmacists' responsibility is to distribute drugs at the window or sell drugs in the pharmacy. The role of pharmacists in the rational use of drugs is ignored. Physicians and nurses also believe that the responsibilities of pharmacists working in fever clinics and isolation wards are just distributing medicines. This may be related to the fact that most hospital pharmacists in China graduated with science degrees (not clinical-based degrees), resulting in a lack of clinical experience. They often encounter difficulties in clinical practice and fail to meet the expectations of physicians, nurses and patients. This implies that hospital pharmacists in China should continue education and acquire a wealth of clinical knowledge for future work transformation. 
Vulnerabilities in drug management discovered by hospital pharmacists

We identified loopholes in managing controlled substances and free drugs. For controlled substances, the following were noted: (1) no real-time or in-person double-checking of controlled substances by pharmacists and nurses, (2) delayed verification of prescriptions since some hospitals still use paper prescriptions for controlled substances, and (3) the proof of destruction of empty ampoules of controlled substances is only presented as photos which could be falsified. Similarly, free drugs, including lopinavir/ritonavir, were not thoroughly managed since it was not easy to trace the drug use. The Chinese government and hospitals are now attempting to draft management plans to use controlled substances, drug retrieval, free drugs and donated drugs during the epidemic. ${ }^{29}{ }^{30}$ On 15 September 2020, the National Health Commission issued a notice on strengthening the management of controlled substances and class I psychotropic drugs in medical institutions, which required improving the entire process for the management of controlled substances to achieve a closed-loop traceability management.

Based on our study findings and the results from previous studies, we propose the following measures to close management loopholes: (1) after electronic medical orders are issued, nurses will call the pharmacy to request the controlled substances. The nurse assists the pharmacist in completing the prescription information for controlled substances, verifying the authority of the prescribing physician, and confirming the physician's signature consistency between the electronically printed prescription and the red paper prescription. The pharmacist then reviews the batch number, dosage, specification, quantity, and drug administration method to ensure the standardised use and safety of controlled substances. This successful measurement has been used by the Department of Pharmacy at Tongji Hospital of Huazhong University of Science and Technology. ${ }^{31}$ (2) Use intelligent equipment such as smart storage cabinets to improve work efficiency and avoid drug diversions. (3) Empty ampoules and waste stickers should be destroyed by two persons in the isolation wards. Video recordings should be stored and registered. (4) Pharmacists should strictly implement the principle of non-return of drugs that require refrigeration or light-protection. (5) For free drugs, pharmacists should formulate a reasonable medication system, maintain communication with physicians and nurses, and strictly record the use of each drug.

\section{The impact of novel coronavirus pneumonia on the future work of pharmacists}

In China, pharmacists have gradually shifted towards a 'patient-centred' work model. Pharmacists actively provide clinical activities to ensure the safe, effective and economical use of drugs. During the COVID-19 outbreak in China, problems have been exposed and have profoundly impacted the future work of pharmacists.
These include: (1) accelerating the process and rules and regulations for standardising drug mailing services and accelerating the development of internet diagnosis and treatment of chronic diseases; (2) promoting the development of telemedicine and at the same time promoting the development of remote pharmaceutical services; (3) promoting the formulation of emergency strategies and conducting actual combat drills for public health incidents to prepare for future accidents; (4) promoting the development of smart pharmacy; and (5) promoting science popularisation activities for rational use of medicines, and improving the level of rational use of medicines for the whole population.

Our study has the following limitations: first, we could only conduct one focused interview session at SPHZ due to strict infection control. However, we identified some loopholes in drug management and gained some insights regarding our study objectives which could be explored further in subsequent semistructured interviews. Second, we only focused on the work experience of the pharmacists during the COVID-19. We did not explore their psychological experiences as several studies have been conducted to investigate healthcare providers' mental health during the COVID-19 pandemic. ${ }^{32}$ Third, our study is limited to one province in China. Subsequent studies should include pharmacists from other regions. Fourth, this manuscript was mainly about the work experience of pharmacists in fever clinics and isolation wards.

\section{SUMMARY}

This qualitative study reveals that pharmacists have played an essential role in the fight against the COVID-19 epidemic in China. The issues identified can guide developing management strategies to better prepare pharmacies and pharmacists to work against future public health crises.

Contributors $\mathrm{JH}, \mathrm{SD}$ and $\mathrm{ZY}$ designed the study. JH carried out the interviews. ZY drafted the manuscript.JH, XJ and XW finished the initial transcription and analysed the data. YY and SD have been involved in drafting and revising the manuscript. All authors read, revised and approved the final manuscript.

Funding This work was supported by the Youth Innovation Fund of the First Affiliated Hospital of Zhengzhou University (grant number not available).

Competing interests None declared.

Patient and public involvement Patients and/or the public were not involved in the design, or conduct, or reporting, or dissemination plans of this research.

\section{Patient consent for publication Not required.}

Ethics approval The First Affiliated Hospital of Zhengzhou University Institutional Review Board approved the study protocol (2020-KY-224). To participate in the study, informed written consent was obtained from the participants. All methods were carried out in accordance with relevant guidelines and regulations.

Provenance and peer review Not commissioned; externally peer reviewed.

Data availability statement Data are available upon reasonable request. Data are available upon reasonable request. The data are the interview transcripts and audio files from the interview of the participants. Data are available from the corresponding authors $\mathrm{ZY}$ and $\mathrm{SD}$ on reasonable request.

Open access This is an open access article distributed in accordance with the Creative Commons Attribution Non Commercial (CC BY-NC 4.0) license, which 
permits others to distribute, remix, adapt, build upon this work non-commercially, and license their derivative works on different terms, provided the original work is properly cited, appropriate credit is given, any changes made indicated, and the use is non-commercial. See: http://creativecommons.org/licenses/by-nc/4.0/.

\section{ORCID iDs}

Jie Hao http://orcid.org/0000-0001-7792-252X

Zhao Yin http://orcid.org/0000-0003-2589-0962

\section{REFERENCES}

1 Zhu N, Zhang D, Wang W, et al. A novel coronavirus from patients with pneumonia in China, 2019. N Engl J Med 2020;382:727-33.

2 Dhama K, Khan S, Tiwari R, et al. Coronavirus disease 2019-COVID-19. Clin Microbiol Rev 2020;33:e00028-20.

3 Velavan TP, Meyer CG. The COVID-19 epidemic. Trop Med Int Health 2020;25:278-80.

4 Rothan HA, Byrareddy SN. The epidemiology and pathogenesis of coronavirus disease (COVID-19) outbreak. J Autoimmun 2020;109:102433.

5 Tu H, Tu S, Gao S, et al. Current epidemiological and clinical features of COVID-19; a global perspective from China. J Infect 2020;81:1-9.

$6 \mathrm{Ge} \mathrm{H}$, Wang X, Yuan X, et al. The epidemiology and clinical information about COVID-19. Eur J Clin Microbiol Infect Dis 2020;39:1011-9.

7 Yu Z, Miao H, Chunhua L. Experience of frontline clinicians fighting against COVID-19: a qualitative study. Chinese General Practice 2020;23:1078-82.

8 Shengjia W, Caihong P, Lei K. Qualititative research on first-line nurses who are fighting back with coronavirus disease 2019 in a three grade Hospital. Chinese Journal of Nursing 2020;55:141-4.

9 Qiong S, Jing G, Xiao L. Qualitative Research of Nurses's Work Experiences on the Front-line of COVID-19 in a Large General Hospital. Medicine and Society 2020;33:76-81.

10 Eftekhar Ardebili M, Naserbakht M, Bernstein C, et al. Healthcare providers experience of working during the COVID-19 pandemic: a qualitative study. Am J Infect Control 2021;49:547-54.

11 Li H, Zheng S, Liu F, et al. Fighting against COVID-19: innovative strategies for clinical pharmacists. Res Social Adm Pharm 2021;17:1813-8.

12 Lange M, Joo S, Couette P-A, et al. Impact on mental health of the COVID-19 outbreak among community pharmacists during the sanitary lockdown period. Ann Pharm Fr 2020;78:459-63.

13 Johnston K, O'Reilly CL, Cooper G, et al. The burden of COVID-19 on pharmacists. J Am Pharm Assoc 2021;61:e61-4.

14 Gill P, Stewart K, Treasure E, et al. Methods of data collection in qualitative research: interviews and focus groups. Br Dent $J$ 2008;204:291-5.

15 Northall T, Chang E, Hatcher D, et al. The application and tailoring of Colaizzi's phenomenological approach in a hospital setting. Nurse Res 2020;28:20-5.

16 Schindel TJ, Yuksel N, Breault R, et al. Perceptions of pharmacists' roles in the era of expanding scopes of practice. Res Social Adm Pharm 2017;13:148-61.
17 Jin $\mathrm{Y}-\mathrm{H}$, Huang $\mathrm{Q}$, Wang $\mathrm{Y}-\mathrm{Y}$, et al. Perceived infection transmission routes, infection control practices, psychosocial changes, and management of COVID-19 infected healthcare workers in a tertiary acute care hospital in Wuhan: a cross-sectional survey. Mil Med Res 2020;7:24.

18 Alves da Costa F, Lee V, Leite SN, et al. Pharmacists reinventing their roles to effectively respond to COVID-19: a global report from the International pharmacists for anticoagulation care Taskforce (iPACT). J Pharm Policy Pract 2020;13:12.

19 Song Z, Hu Y, Zheng S, et al. Hospital pharmacists' pharmaceutical care for hospitalized patients with COVID-19: recommendations and guidance from clinical experience. Res Social Adm Pharm 2021;17:2027-31.

20 Ying W, Qian Y, Kun Z. Drugs supply and pharmaceutical care management practices at a designated Hospital during the COVID-19 epidemic. Res Social Adm Pharm 2021;17:1978-83.

21 Meng L, Huang J, Qiu F, et al. Roles of the Chinese clinica pharmacist during the COVID-19 pandemic. J Am Coll Clin Pharm 2020;38. doi:10.1002/jac5.1274. [Epub ahead of print: 04 Jun 2020].

22 Elbeddini A, Prabaharan T, Almasalkhi S, et al. Pharmacists and COVID-19. J Pharm Policy Pract 2020;13:36.

23 Bell JS, Reynolds L, Freeman C, et al. Strategies to promote access to medications during the COVID-19 pandemic. Aust J Gen Pract 2020;49:530-2.

24 Margusino-Framiñán L, Illarro-Uranga A, Lorenzo-Lorenzo K, et al. Pharmaceutical care to hospital outpatients during the COVID-19 pandemic. Telepharmacy. Farm Hosp 2020;44:61-5.

25 Bauman JL. Hero clinical pharmacists and the COVID-19 pandemic: Overworked and overlooked. J Am Coll Clin Pharm 202010.1002 jac5.1246. [Epub ahead of print: 05 May 2020].

26 Fangfang L, Li D, Peng S. Research on the Work Status Quo and Countermeasures of Pharmacists in Public Hospitals of Chongqing under the Background of "New Medical Reform". China Pharmacy 2019;30:3350-4.

27 Lizheng $\mathrm{C}$. The difficulties and strategies of primary pharmacists in developing pharmaceutical service. Strait Pharmaceutical Journal 2010;22:195-6.

28 Jing $\mathrm{H}$, Shimin $\mathrm{Y}$. The present situation, existing problems and development suggestions of clinical pharmacists in China. China Pharmacy 2004:6-8.

29 Shao L, Gefei H, Jie D. Pharmaceutical emergency guarantee difficulties and countermeasures for the prevention and control of outbreak of Corona Virus Disease 2019(COVID-19). Chinese Journal of Hospital Pharmacy 2020;40:243-9.

30 Xiang-yi S, Peng-xiang Z, Li Y. Establishment and practice of emergency management plan for hospital fever pharmacy under coronavirus disease 2019 epidemic. J Clin Med 2020;18:89-92.

$31 \mathrm{Li} \mathrm{C}$, Minghui S. Practice and reflections on the management of narcotic drugs during the COVID-19 pandemic. Herald of Medicine 2020;39:1051-3.

32 Fang M, Xia B, Tian T, et al. Drivers and mediators of healthcare workers' anxiety in one of the most affected hospitals by COVID-19: a qualitative analysis. BMJ Open 2021;11:e045048. 